\title{
The LEST technique: Treatment of prostatic obstruction preserving antegrade ejaculation in patients with benign prostatic hyperplasia
}

\author{
Rosario Leonardi \\ Department of Urology and Andrological Surgery, Musumeci GECAS Clinic Gravina of Catania, Catania, Italy.
}

\begin{abstract}
Summary The objective of this study was to search for an alternative technique to relieve prostatic obstruction due to benign prostatic hyperplasia without affecting the ejaculatory function.

The technical requirements are a laser with a wavelength well absorbed by water (good vaporizing effect) and at the same time by hemoglobin (good hemostatic effect) and laser fibers very resistant at high emission power allowing perfect vaporization in a contact mode. The aim of the technique is to avoid damage of the structures that allow the peripheral region of the prostate and the seminal vesicles to discharge their secretions into the posterior urethra. The orifices of the ejaculatory ducts must therefore be identified and preserved, damage of the ejaculatory ducts along their path inside the prostate must be avoided and the so called "genital sphincter" must be saved. The steps of the Leonardi Ejaculation Sparing Technique (LEST) procedure are as follow: Step 1 - ejaculatory duct orifices must be identified and the limits of the vaporization section must be marked. Step 2 - bladder neck is cleaned of the prostate hypertrophic tissue saving, as much as possible, the smooth muscle fibers of the bladder neck. Step 3 - vapo-resection of the lateral lobe (or enucleation of the adenoma) is performed. Step 4 - cautious and meticulous preparation of the prostatic apexes is obtained with saving of the orifices of the ejaculatory ducts. An antegrade ejaculation is maintained in about $80 \%$ of cases in patients without a middle lobe, although in the presence of a middle lobe this rate drops to about $50 \%$.
\end{abstract}

KEY WORDS: Benign prostatic hyperplasia; Prostatic obstruction; Antegrade ejaculation; Laser.

Submitted 25 January 2019; Accepted 8 February 2019

\section{INTRODUCTION}

There is a growing interest to maintain a normal sexual function, after surgical management of bladder outflow obstruction due to benign prostatic hyperplasia (BPO).

It is well known, that transurethral resection of the prostate (TURP) strongly impacts on sexual function, not so much in terms of erection but, of course, in terms of antegrade emission of seminal fluid.

This has led, in recent years, to the search for alternative techniques to relieve obstruction capable of preserving the ejaculatory function.

Unfortunately, most of these techniques had a limited diffusion due to both restricted indications (1) and reduced efficacy of obstruction removal.
In this paper we will describe the technique developed by our team, starting from the anatomical and functional premises that support it.

Furthermore, the process of the development of the technique over time will be reviewed and finally the surgical procedure aiming to achieve a complete removal of bladder neck obstruction with preservation of ejaculatory function will be described "step by step".

\section{Anatomy of the prostate}

The prostate is a glandular and stromal organ, single and median, located in the small pelvis between the base of the bladder and the urogenital diaphragm, behind the pubic symphysis and in front of the rectum.

The first tract of the urethra, namely the prostate urethra, passes through the prostate from the base to its apex. The secretions of the prostate glands, seminal vesicles and testicles (corpuscular part of the seminal fluid) are voided into the lumen of the prostate urethra.

The seminal fluid is made up for about $15-30 \%$ from prostate secretion, for about $50-70 \%$ from the fluids of the seminal vesicles and for less than $5 \%$ from the product of the testicles, deferential ampules, glands of Cowper and urethral glands (2).

The prostate is typically described as a chestnut shape with the base facing the bladder and the apex at the bottom, resting on the external urethral sphincter (3).

Histologically, it is composed of tubulo-alveolar glands whose ducts open into the prostate urethra. The gland can be clinically divided into the anterior lobe, the middle lobe, if present, and two lateral lobes.

McNeal observed that the urethra separates the prostate into two regions, the ventral (fibromuscular) and the dorsal (glandular) one.

Approximately halfway between the apex and the base, the posterior wall of the urethra undergoes an acute angle (ventral angle of 35 degrees), which separates the urethra into a proximal segment and a distal segment.

The orifices of the ejaculatory ducts and the verum montanum are located in the context of the distal segment. The glandular prostate can be divided into four distinct regions: peripheral zone, central zone, transition zone, and periurethral region (4) (Figures 1, 2).

In absence of prostatic hyperplasia (BPH), the peripheral zone makes up about $70 \%$ of the gland. The transition 
Figure 1.

The figure shows schematically a sagittal section of prostate.

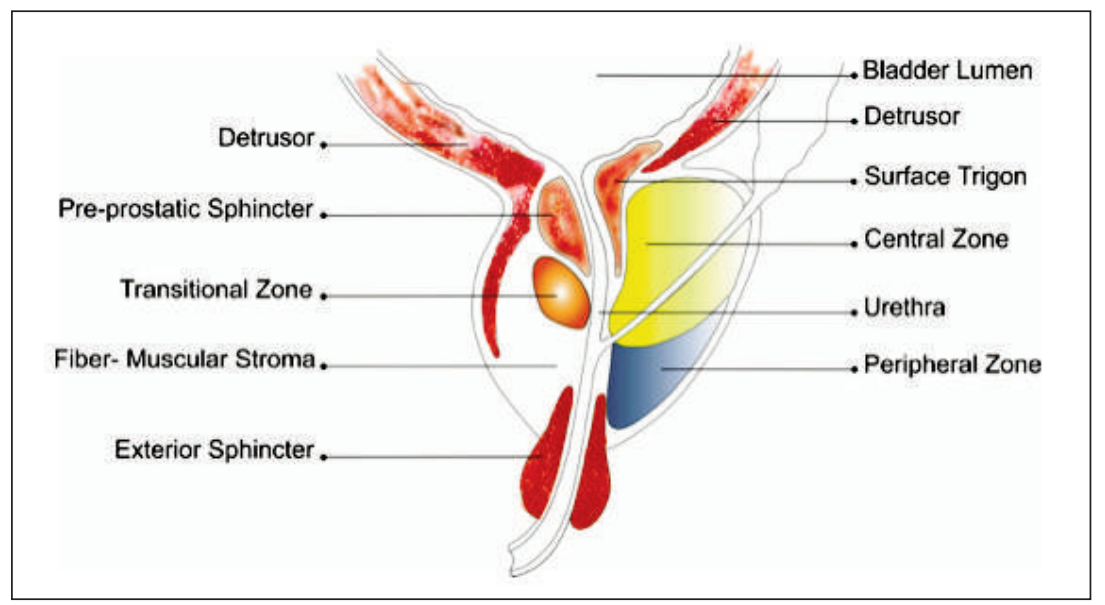

Figure 2.

3D reconstruction of different zones of prostate.

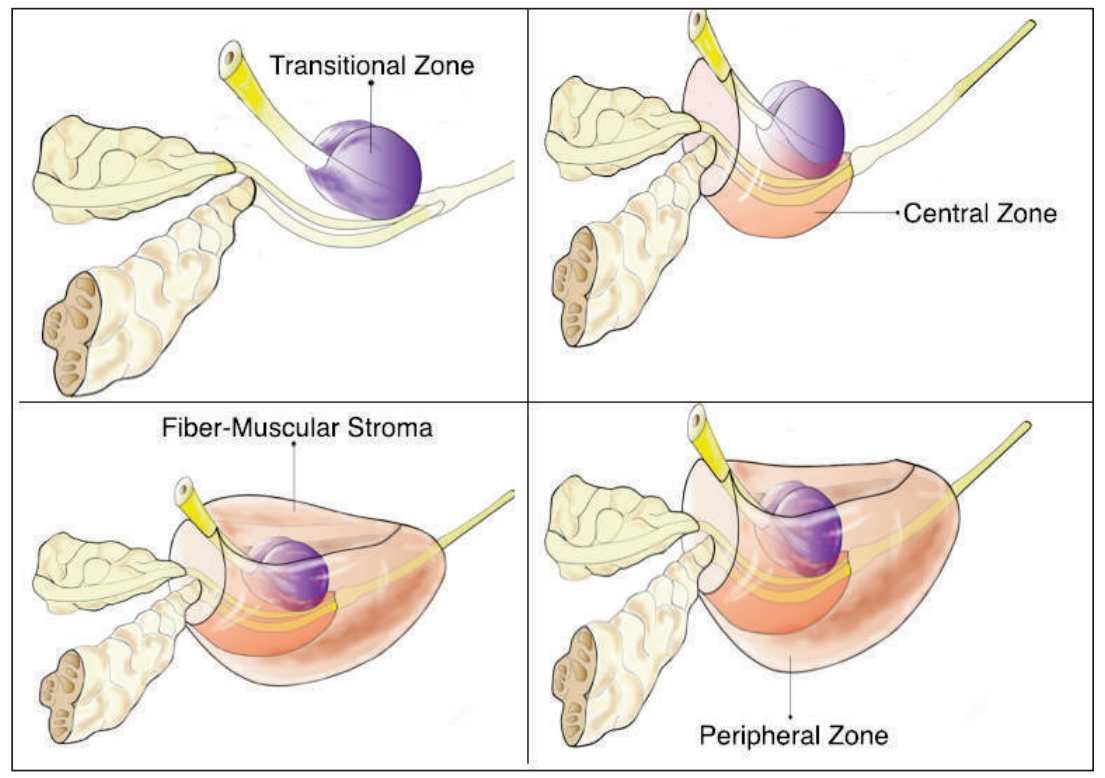

urethra. This phenomenon creates the unstoppable sensation of ejaculation. The seminal fluid injected into the posterior urethra would drain into the bladder if an "anti-reflux" system was not activated by the alpha sympathetic system. Initially, this anti-reflux system was exclusively identified with the bladder neck, but subsequently the histochemical studies of Gosling et Thompson identified this sphincter area in the pre-prostatic segment of the posterior urethra above the veru (6). Bruschini et Tanagho confirmed, by pharmacological and manometric studies, the presence of this area called "genital sphincter" (7) (Figures 3, 4).

This functional sphincter with mainly genital function begins in the bladder neck, continues with the smooth muscle fibers intrinsic to the urethra and is added to the urinary sphincter fibers incorporated in the prostate stroma and to the smooth muscle of prostate glandular elements (8).

To summarize, it is a smooth muscle complex located in the area proximal to the opening of the ejaculatory ducts in the urethra.

These muscles, in the phase in which the action of the sympathetic nervous system prevails, contracts preventing the reflux of the seminal fluid into the bladder (9).

In case of BPH, the transition zone is larger than in the normal prostate. The transitional zone compresses the peripheral zone moving it down its apical portion.

In addition, in 36 to $70 \%$ of patients with $\mathrm{BPH}$ there is a reduction or

zone represents about $5-10 \%$ of the glandular tissue and is located at the angle that divides the tract of the urethra above and below the veru montanum. The central area surrounds and protects the ejaculatory ducts and constitutes about $25 \%$ of the glandular tissue. The fibromuscular zone that does not contain glandular tissue may, in some subjects, constitute about one third of the glandular volume (5).

\section{Functional anatomy of the ejaculation}

Ejaculation consists of three physiological phases.

The first phase, also known as the preparation phase, is under the prevalent influence of the parasympathetic system with an increase in the secretory activity of the prostate glands and seminal vesicles. This is followed by a second phase, called emission phase, which culminates with a coordinated and peristaltic contraction of the smooth musculature of the vasa, ampoules, seminal vesicles and acinar prostatic glands. This results in the emission and accumulation of seminal fluid in the posterior absence of ejaculate, which etiopathogenesis is still unclear. Some authors argue that there may be a compression of the ejaculatory ducts, others that there may be an increase in the angle of the ducts in the terminal portion near their outlet into the urethra. The fact is that the glandular structure of the hypertrophic prostate (transition zone) loses its physiological structure, and the glands, overcrowded and newly formed, often do not produce an adequate volume of secretion compared to a normal gland (Figure 5).

Histological studies with quantitative imaging analysis of prostate sections of symptomatic BPH subjects stained with double enzyme immunoassay showed that, for each area examined, the percentage density of smooth muscle and connective tissue was significantly higher than those of glandular epithelium and glandular lumen area (medium SEM) (10).

From this finding it can be deducted that the prostate component of the seminal fluid, in the subject with BPH, is significantly reduced. 


\section{Figure 3.}

Glandular section along the urinary axis

$F$ Anterior fibromuscular layer; BN Bladder Neck; PZ Peripheral zone; GS Genital Sphincter surrounding the premontanal urethra, ensuring with its contraction, during ejaculation, the orthodromic direction of the seminal flow. Modified from F. Basile et al. Manual of Semeiotics and Surgical Methodology. Edra Publisher 15 Jan 2014.
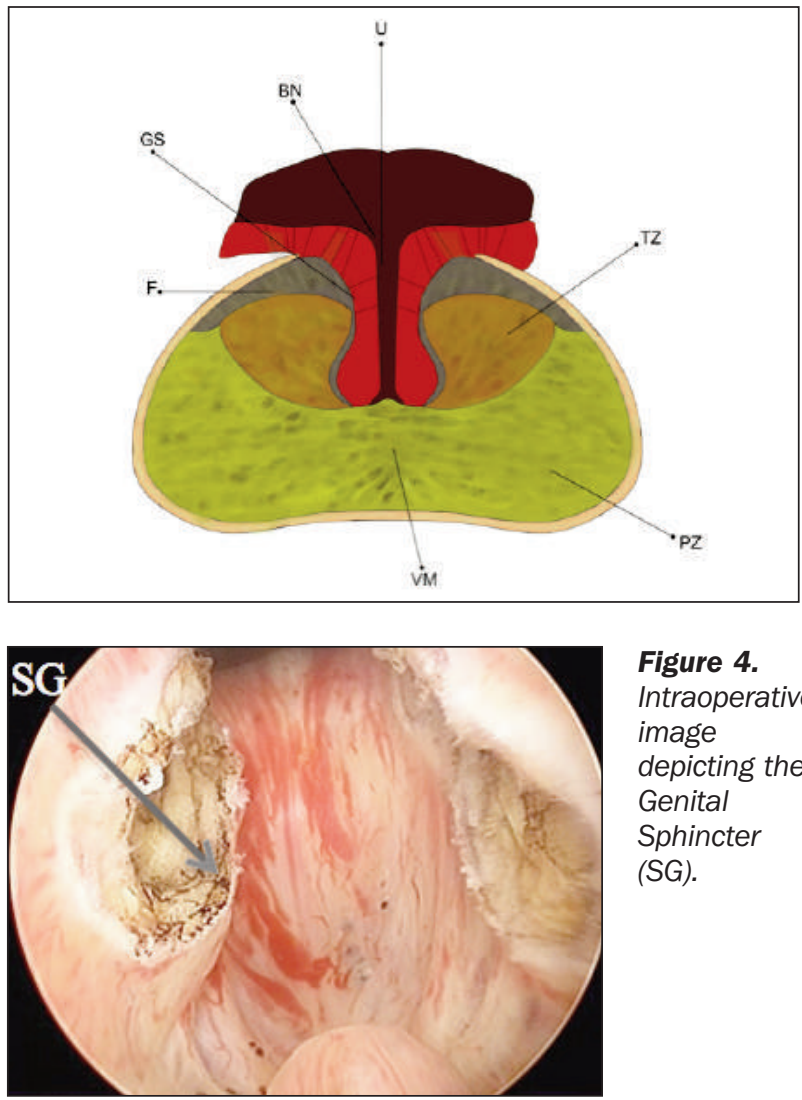

Figure 4. Intraoperative image depicting the Genital Sphincter (SG).

Figure 5.

Histological appearance of prostate glandular tissue in a normal subject and in a subject with benign prostatic hyperplasia.

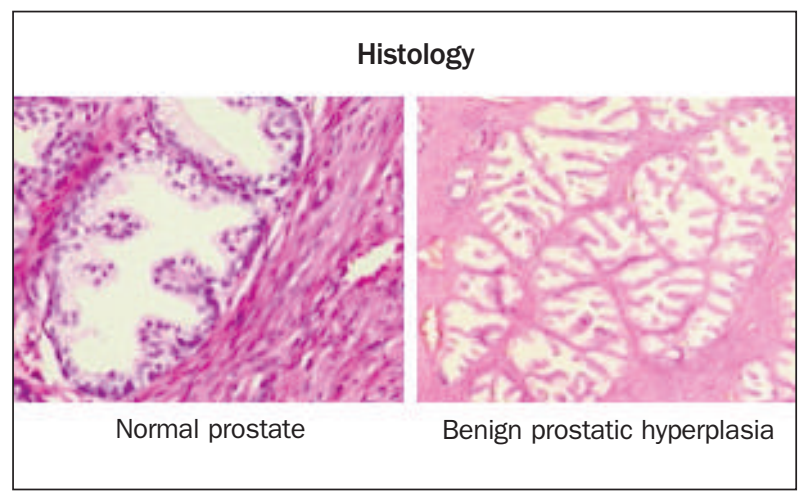

\section{LEST procedure}

\section{(Leonardi Ejaculation Sparing Technique)}

As mentioned in the introduction, the development of the LEST technique originates from the growing demand from the male population to be able to maintain an intact sexual function (erection and ejaculation) after an efficacious procedure of removal of bladder neck obstruction (11). Although the gold standard of endoscopic surgical treatment for benign prostatic hyperplasia (BPH) has been, until now, the transurethral resection of the prostate (TURP), in recent years the supremacy of this procedure has been challenged by the introduction of new surgical devices and, above all, of endoscopic lasers capable of removing the prostate adenoma, that is the cause of cervical-urethral obstruction. These techniques have, in fact, reduced hospitalization times and the complications associated with TURP (hemorrhage, incontinence) (12). Until now, little attention was given to the maintenance of ejaculatory function, considering retrograde ejaculation as a minor side effect for the quality of life of the patient, if not as a tangible sign of a successful removal of obstruction.

However, patients, if accurately interviewed, do not tolerate retrograde ejaculation, and, for many of them, this risk is the only reason that leads them to delay the procedure, even when mandatory, for fear of incurring this post-operative outcome $(13,14)$.

We have therefore decided, on the basis of the anatomical-functional premises described above, to develop the LEST technique, whose preliminary data have already been published in 2009 (15). We believed that a complete removal of obstruction, overlapping to that achievable by a successful TURP, should not necessarily result in a retrograde ejaculation. This could be possible because the removal of hypertrophic tissue, as mentioned above, will reduce the volume of semen only by a small amount, since hypertrophic tissue produces a reduced amount of secretions. In fact the most volume of semen, especially in subjects with BPH, comes from the glands of the peripheral zone and seminal vesicles.

It was therefore necessary:

1. To conceive a technique that would not damage the structures that allow these anatomical regions to introduce their secretions into the posterior urethra. Secretions of the peripheral region of the prostate and the seminal vesicles discharge their secretions at the base of the prostatic urethra above the veru (pre-montonal) through the orifices located in the region surrounding the veru montanum. The orifices of the ejaculatory ducts must therefore be identified and preserved.

2. To avoid damaging the ejaculatory ducts along their path inside the prostate, remembering that they are surrounded by the central part of the prostate and that they get closer and closer to the floor of the prostate urethra, as they approach their openings.

3. To save what Tanagho, and after him other authors, called "genital sphincter", as previously described in the anatomical-functional premises.

4. To have the equipment adequate to develop an unobstructed prostatic lodge without damaging all the structures above described.

\section{Lasers}

In relation to this latter point we had to exclude the devices used for traditional resection, aiming to find a laser with a suitable wavelength to be well absorbed by water (good vaporizing effect), and at the same time by hemoglobin (good hemostatic effect) (Figure 6). It was also necessary to 


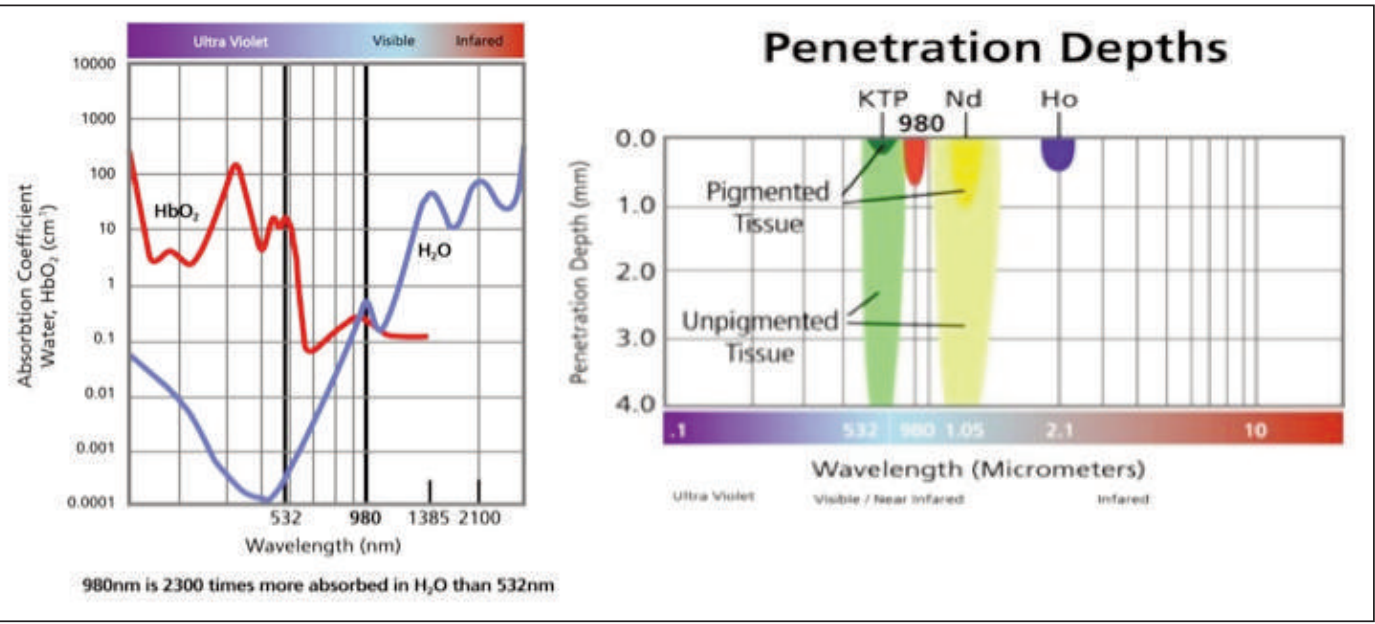

Figure 6.

The figure shows at the left the absorption spectrum at wavelength 980 $\mathrm{nm}$ in water and hemoglobin, at the right the depth of penetration in the prostate tissue of 980 and other types of laser. choose a wavelength that did not have a significant effect of deep penetration into the tissues producing only a surface vaporization. This is to avoid the very annoying irritative symptoms due to the reabsorption of the coagulative necrosis produced in depth of the unremoved prostate tissue that was associated with the use of lasers already present on the market. The first laser we tried, able to have all the requirements above described, was a laser with 980 $\mathrm{mm}$ wavelength working at the power of 100 watts. Since 2010 a significant improvement was achieved by the use of the new HPD 180 watts high power laser, and, by the production of fibers designed and built to work in contact and at high power. Another improvement was done in 2016 with a new laser that is able to mix freely two wave lengths (980 $\mathrm{nm}$ and $1470 \mathrm{~nm}$ ). This diode laser is capable of emitting two wavelengths of $980 \mathrm{~nm}$ and $1470 \mathrm{~nm}$ simultaneously along the same fiber. In practice it is possible to mix, according to the structure of the tissue to be vaporized, a prevalence of $980 \mathrm{~nm}$ with a stronger affinity for hemoglobin or $1470 \mathrm{~nm}$ with a higher absorption in water for less vascularized tissues.

The low penetration of both wavelengths into the vaporized tissue (350 microns) (18) allows not only to reduce post-operative irritative symptoms but, at the same time, to safeguard the ejaculatory ducts.

\section{Fibers}

From the very first attempts we realized the importance of using contact vaporization. The vaporization for irradiation, produced by the "side-firing fibers", is not able to sculpt the prostatic lodge with millimetric precision. The beam of light emitted by the fiber can create a more or less extended vaporization margin, depending on the distance of the emitting fiber from the prostate tissue. With the "side-firing fiber" a good vaporization is obtained keeping, theoretically, the fiber at a constant distance of $0.5 \mathrm{~cm}$ from the tissue to be vaporized. If this distance is not maintained, the radiation power on the tissue is reduced, creating coagulative necrosis instead of vaporization. In our previously published study we did not dispose of "contact" vaporizing fibers, so we had to use a trick that allowed us to use the side fibers in a contact mode. We set the laser to pulsed mode in order to reduce the stress on the fiber by allowing some cooling in the refractory phase of emission. The results obtained in the previous study were satisfactory in terms of removal of obstruction and maintaining the anterograde ejaculation (Table 1). Now we are using new fibers called Twister of three sizes (Normal, Large and Extra Large) (16) (Figure 7).

The Twister fiber is a special fiber with a quartz tip, with a $15^{\circ}$ curvature at the tip, which allows perfect vaporiza-

\section{Table 1.}

Results of "side-firing" laser. Modified by Leonardi R.:

Preliminary results on selective light vaporization with the sidefiring $980 \mathrm{~nm}$ diode laser in benign prostatic hyperplasia: an ejaculation sparing technique. Prostate Cancer Prostatic Dis. 2009; 12:277-80.

\begin{tabular}{|lccc|}
\hline Parameter & Baseline $\mathbf{n}=\mathbf{5 2}$ & 3-month $\mathbf{n}=\mathbf{5 2}$ & 12-month $\mathbf{n}=\mathbf{2 2}$ \\
\hline IPSS & $18.4+/-5.8$ & $7.5+/-5.9$ & $6.0+/-0.6$ \\
\hline IPSS improvement \% & & $-59 \%$ & $-67 \%$ \\
\hline$Q_{\max } \mathrm{ml} / \mathrm{sec}$ & $7.5+/-4.1$ & $20.9+/-8.4$ & $19.7+/-1.4$ \\
\hline$Q_{\max }$ improvement \% & & $+179 \%$ & $+163 \%$ \\
\hline PVR $\mathrm{ml}$ & $160+/-140$ & $24+/-22$ & $20.3+/-4.4$ \\
\hline PVR improvement \% & & $-85 \%$ & $-87 \%$ \\
\hline IPSS-QoL & $3.5+/-1.2$ & $1.3+/-1.2$ & $1.2+/-0.4$ \\
\hline & $-63 \%$ & $-66 \%$ \\
\hline $\begin{array}{l}\text { IPSS }=\text { International Prostate Symptom Score } \\
\text { PVR }=\text { Postvoidal residual urine } \\
\mathbf{Q}_{\max }=\text { Maximum flow rate } \\
\text { QoL }=\text { Quality of Life } \\
\text { Change from baseline for all parameters } P<0.0001\end{array}$ \\
\hline
\end{tabular}

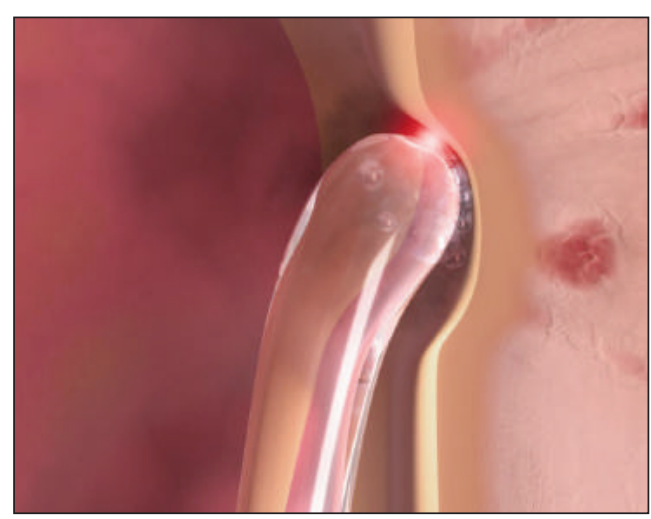

Figure 7.

Twister

Fiber XL. 
Figure 8.

Ejaculatory triangle.

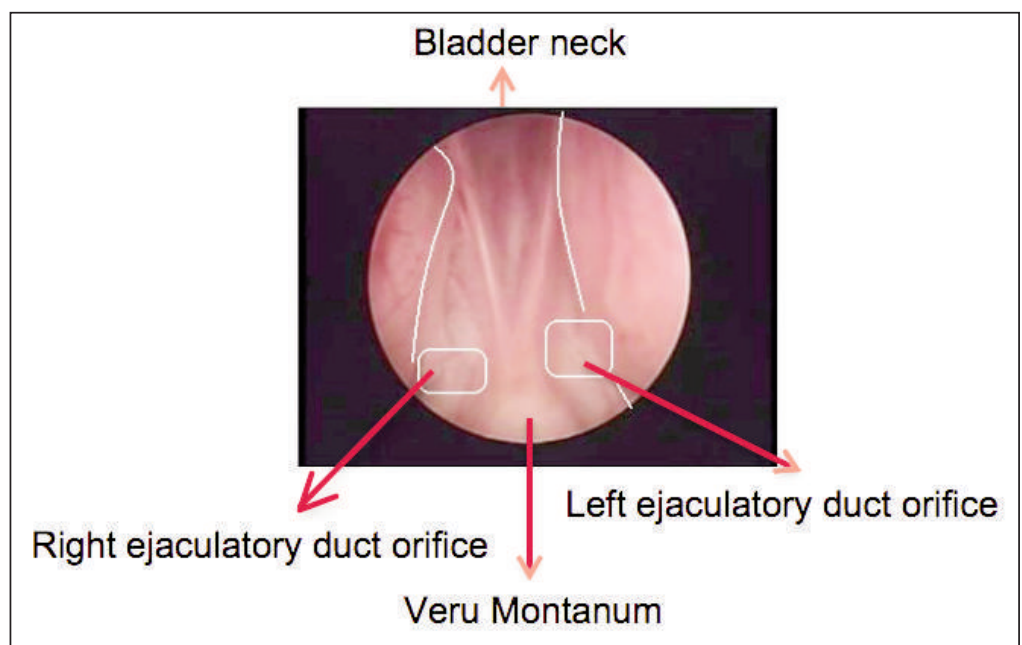

a middle lobe, the rate drops to about 50\%. The ideal feature of the patient for LEST surgery is a bilobed prostate with a good percentage of peripheral prostate, well visible ejaculatory duct orifices (ODEs) and normal seminal vesicles. For this reason, we always practice a trans-rectal prostate ultrasound and flexible urethroscopy on our patients before surgery. The worst results are in prostate with voluminous medium lobe, non-visible ODEs and hypotrophic seminal vesicles.

Why most suitable prostates for LEST should not have a middle lobe?

We previously described which anatomical structures are responsible for the persistence of antegrade ejaculation, namely the smooth muscles that extends from the bladder neck along the first tract of the posterior urethra to the veru montanum.

As we know, in the trilobal prostates, the middle lobe plays an important role in gen-

tion and/or engraving of the tissue, in a contact mode. The three measures are distinguished by the volume of the quartz tip and the measure has to be chosen according to the prostate volume that must be vaporized. The Twister Fiber is very resistant even when used at high emission powers, and allow, in almost all of cases, to conclude the procedure with a single fiber (17).

These technological innovations have allowed us to be much faster in prostate vaporization, reaching a real speed of tissue removal of about 1.5 grams per minute. The indications have been extended to the treatment of increasingly voluminous prostates, but also allowed us to precisely sculpt the new prostate loggia with excellent control of the vaporization margins and, therefore, with a saving of the structures responsible for the anterograde ejaculation, that was crucial for developing the LEST technique. The area to be preserved has been named by us as the "ejaculatory triangle" (Figures 8, 9).

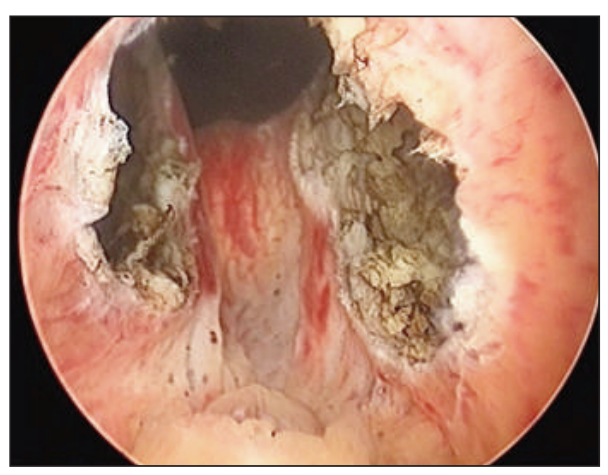

Figure 9. Ejaculatory triangle at the end of the procedure.

\section{Surgical technique}

The patient who requires a LEST procedure must be well informed about the technique that we are going to do, knowing well which are the success rates of the "ejaculation sparing" technique, in order not to feed false expectations. Studies conducted by us have shown the possibility of maintaining an anterograde ejaculation in about $80 \%$ of cases in patients without a middle lobe. In the presence of erating a severe obstruction and therefore, for a successful removal of obstruction, it is necessary to remove all the medium lobe from its origin, near to the veru montanum. This involves the removal of most of the ejaculatory triangle and a weakening of the bladder neck. This is the reason, in our opinion, why the presence of a voluminous middle lobe lowers the success rate of the intervention to about $50 \%$.

Why is a good presence of peripheral prostate important? By removing most of the transition zone (reaching the surgical capsule), after the procedure prostate secretion is limited to the peripheral region, which pours its secretion on the floor of the prostate urethra (ejaculatory triangle). As far as seminal vesicles are concerned, we know that, under normal conditions, these structures contribute to producing about $65 \%$ of the volume of seminal fluid.

Having, therefore, two normotrophic seminal vesicles at ultrasound examination and two ODE clearly visible at endoscopic examination, we can predict a good result in term of volume of liquid produced in the post-operative.

\section{Procedure step by STeP}

Step 1: Identification of anatomical structures

Introduced the laser cystoscope with a $30^{\circ}$ optic, the ODEs are identified and the limits of the vaporization section are marked (Figure 10).

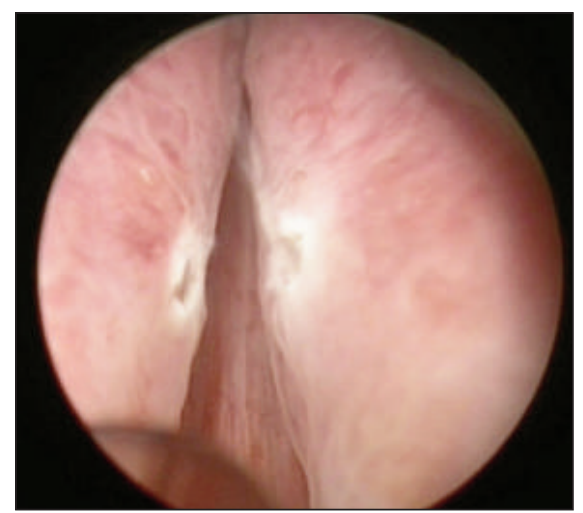

Figure 10.

Markers of the lower limit of the vaporesection. 


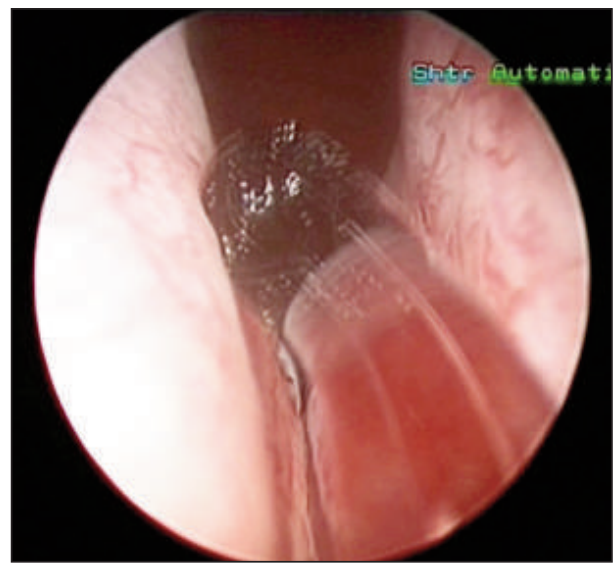

Figure 11. Start of vapo-resection from the bladder neck.

Figure 12.

Bladder neck after surgery. Note the bloodless field, the well defined margin of vapo-resection with sparing of the neck and bladder mucosa that, when washing is stopped, prolapses inside the prostate neo lodge.

\section{Bladder neck after Laser procedure}

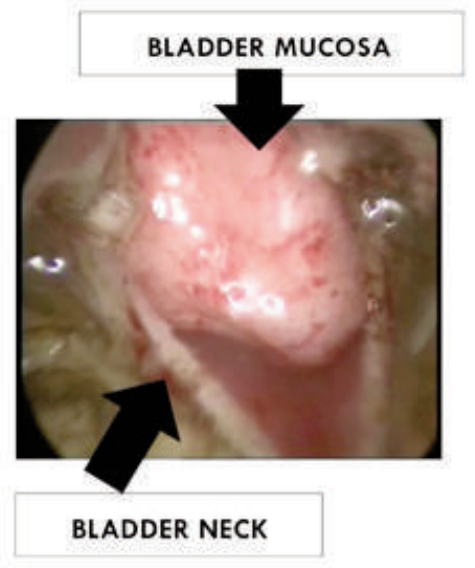

\section{Step 2: Bladder neck preparation}

The vaporization starts with a power lower than the maximum usable for the type of fiber used, in order to avoid thermal shocks to the fiber itself. The bladder neck is cleaned of the prostate hypertrophic tissue that caused the obstruction, saving, as much as possible, the smooth muscle fibers of the neck and ensuring that the neck does not remain rigid after vaporization. We proceed with small incisions at 5 and 7 o'clock, taking care not to destroy the muscle fibers completely. It is always important to identify and preserve the bladder neck fibers. At the end of the vaporization, a clean cut is observed between the bladder mucosa and the prostate lodge (Figures 11, 12).

\section{Step 3: Vapo-resection of the lateral lobes}

Contact vaporization must always meet the criteria of symmetry. The prostatic lodge after removal of obstruction must be large and symmetrical.

We start vapo-resection at the base of the lateral lobes so that the edges of the ejaculatory triangle are clearly defined. It should be noted that, in larger prostates, and if it is considered necessary to obtain abundant tissue to be subjected to histological examination, instead of carrying out a vapo-resection, a real enucleation of the adenoma (DiLEP) can be used. In these cases, enucleation is performed sparing the structures considered crucial to obtain an antegrade ejaculation (Figure 13).

This last step is perhaps the most delicate and important for the success of the procedure (Figures 14, 15).

The prostate apexes with the traditional TURP are completely removed, believing that, in this way, the maximum removal of obstruction will be obtained. In fact, anatomical studies have shown that the prostate apex is only partly constituted by the transitional tissue causing the obstruction. Part of the apexes are constituted by the peripheral area of the gland that has been moved down by the hypertrophy of transitional zone.

A proof of this is that, at times, some prostate tumors, which are known to develop more frequently in the

\section{Figure 13.}

Final result after DiLEP with LEST technique. On the left trans-rectal ultrasound examination showing the large lodge with preservation of the neck. In the small box, endoscopic image of preservation of part of the apexes belonging to the peripheral zone of the gland. On the right endoscopic image showing preservation of the floor of the prostate urethra where the peripheral gland flow into.

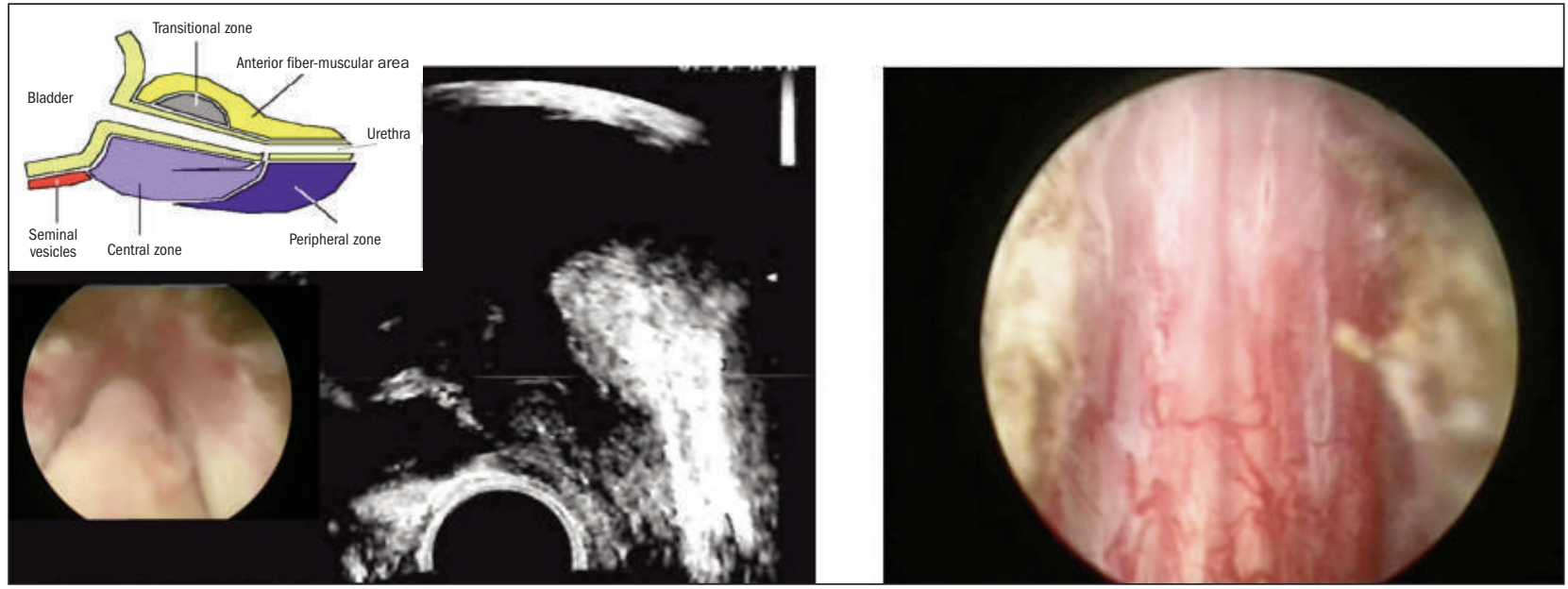




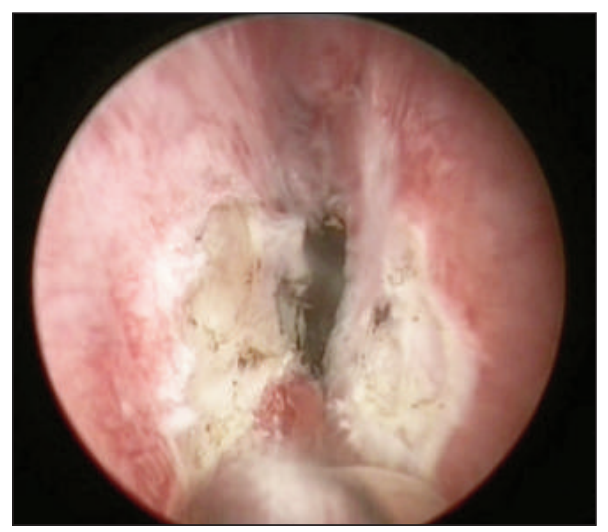

Figure 14.

Start of the vapo-resection of the apexes.

Figure 15.

Trans-rectal ultrasound image after DiLEP with LEST technique.

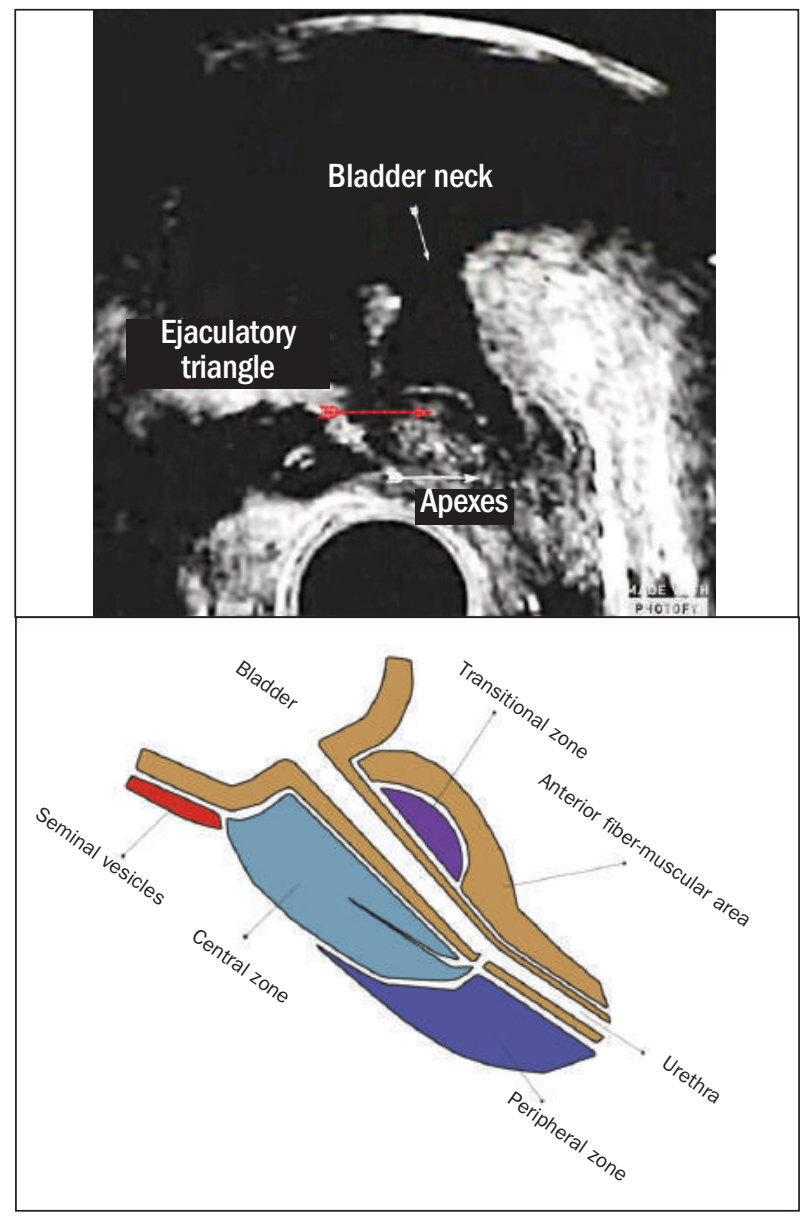

peripheral zone but not in the transition zone, are found in the biopsies of the apex.

The peripheral part of the prostate tissue does not have a structure that could obstruct the urinary flow. Its preservation guarantees the integrity of the genital sphincter and protects the outlets of the ejaculatory ducts during the vaporization phase of this area.

Another fundamental step for the success of the procedure is the preservation of the orifices of the ejaculatory ducts. In doing so, we ensure that the secretions from the seminal vesicles are introduced into the posterior urethra (Figure 16).

At this point the intervention can be considered completed.

We administer an alpha-blocker in the first 20 days of the post-operative. The choice to administer the alphablocker is to avoid spasms of the smooth muscles of the bladder neck which, with the LEST technique, is spared. We do not administer cortisone or painkillers because treatment with the above technique and with wavelengths 980/1470 does not produce significant irritative symptoms. The patient can resume normal sexual intercourse 20 days after surgery.

\section{REFERENCES}

1. Hoepffner JL, Fontaine E, Benfadel S, et al. A modified cervicoprostatic incision technique in hypertrophic adenoma in young subjects desiring to preserve ejaculation. Prog Urol. 1994; 4:371-7.

2. Segawa A. Studies on secretory function of male sexual organs and their accessory glands. Jap J Urol. 1957; 48:869.

3. Seisen T, Rouprêt M, Faix A, Droupy S. The prostate gland: a crossroad between the urinary and the seminal tracts. Prog Urol. 2012; 22 (Suppl 1):S2-6.

4. Selman SH. The McNeal prostate: a review. Urology. 2011; 78:1224-8.

5. Basile F, Bellantone R, Biondi A, et al. Manuale di Semeiotica e Metodologia Chirurgica, EDRA, 2014; ISBN: 9788821434365.

6. Gosling JA, Thompson SA. A neurohistochemical and histological study of peripheral autonomic neurons of the human bladder neck and prostate. Urol Int. 1977; 32:269-76.

7. Bruschini H, Schmidt RA, Tanagho EA. The male genitourinary sphincter mechanism in the dog. Invest Urol. 1978; 15:284-7.

8. Belgrano E, et al. I disturbi dell'eiaculazione Edizioni Medico Scientifiche, Pavia 1995.

\section{EJACULATORY DUCTS OUTLETS}

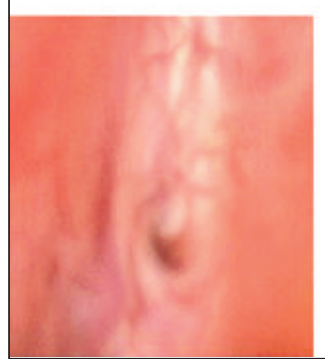

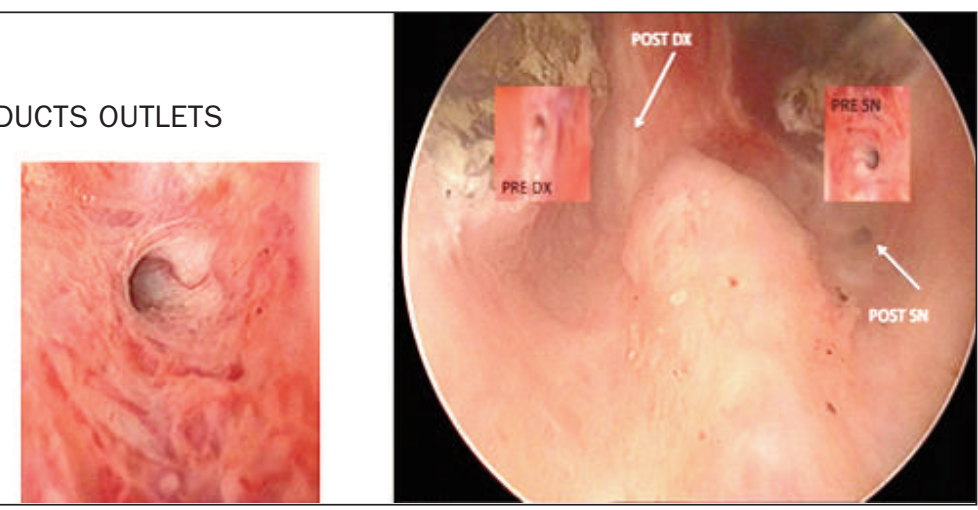

Figure 16.

Pre- and post-surgery orifices of the learned ejaculators. 
9. Stockamp K, Schreiter F. Proceedings: Function of the posterior urethra in ejaculation and its importance for urine control. Urol Int. 1974; 29:226-30.

10. Shapiro E, Hartano V, Lepor V. Anti-desmin vs. anti-actin for quantifying the area density of prostate smooth muscle. Prostate. 1992; 20:259-67.

11. Chung A, Woo HH. Preservation of sexual function when relieving benign prostatic obstruction surgically: can a trade-off be considered? Curr Opin Urol. 2016; 26:42-8.

12. Wang L, Yu QY, Liu Y, et al. Efficacy and safety of laser surgery and transurethral resection of the prostate for treating benign prostate hyperplasia: a network meta-analysis.Asian Pac J Cancer Prev. 2016; 17:4281-4288.

13. Alwaal A, Breyer BN, Lue TF. Normal male sexual function: emphasis on orgasm and ejaculation. Fertil Steril. 2015; 104:1051-60.

14. Lue $T$, et al. Sexual dysfunctions in men health clinical.
Manual of Sexual Medicine Health Publications Ltd. 2004 ISBN 09546956-1-5.

15. Leonardi R. Preliminary results on selective light vaporization with the side- firing $980 \mathrm{~nm}$ diode laser in benign prostatic hyperplasia: an ejaculation sparing technique. Prostate Cancer Prostatic Dis. 2009; 12:277-80.

16. Shaker HS, Shoeb MS, Yassin MM, Shaker SH. Quartz head contact laser fiber: a novel fiber for laser ablation of the prostate using the $980 \mathrm{~nm}$ high power diode laser. J Urol. 2012; 187:575-9.

17. Shaker H, Alokda A, Mahmoud H. The Twister laser fiber degradation and tissue ablation capability during 980-nm highpower diode laser ablation of the prostate. A randomized study versus the standard side-firing fiber. Lasers Med Sci. 2012; 27:959-63.

18. Leonardi R, Caltabiano R, Lanzafame S. Histological evaluation of prostatic tissue following transurethral laser resection (TULaR) using the $980 \mathrm{~nm}$ diode laser. Arch Ital Urol Androl. 2010; 82:1-4.

\section{Correspondence}

Rosario Leonardi, MD (Corresponding Author)

leonardi.r@tiscali.it

Department of Urology and Andrological Surgery,

Musumeci GECAS Clinic Gravina of Catania, Catania (Italy) 